\title{
Dynamical Stellar Dust Shells: Different Perspectives
}

\author{
E. Sedlmayr
}

Zentrum für Astronomie und Astrophysik, TU Berlin, Hardenbergstraße 36, D-10623 Berlin, Germany; e-mail:

sedlmayr@astro.physik.tu-berlin.de

\section{Introduction}

In the last decades considerable progress has been achieved in the physical understanding and modelling of dusty stellar atmospheres and surroundings, as represented by stationary dust driven winds, pulsating shells of Miras and LPVs, episodic phenomena, like occultations of RCrB stars, the atmospheres of brown dwarfs, or even by "hot" objects like dust forming Wolf-Rayet stars.

For all these systems the notions "understanding" and "modelling" depend on the level of the physical approach defined by specific perspectives chosen by a basic "window of perception" for each class of particular objects and its appropriate description within this frame. This is defined by the fundamental assumptions with regard to the global and local appearance and the properties of the considered object, its focused characteristic length and time scales of the various determining processes taken into account, and, of course, by an appropriate body of physical equations necessary for an adequate description within the context of the adopted perspective. In this view, any real approach concentrates on special aspects (or a combination of it), like radiative transfer, wind generation, spectral appearance, dynamical behaviour, global and local stability, cluster nucleation, grain growth and processing, chemistry, interplay of physical and chemical processes, etc. Consequently, any approach projects only particular properties of the considered object, each constituting important ingredients necessary for a final consistent quantitative modelling and a comprehensive physical understanding.

Within such a broad scope, the theoretical problem of dusty objects comprises in principle a variety of very different approaches considered as building blocks of a final realistic qualitative and quantitative description of the overall system and its detailed actual local and global structures. Due to its inherent complexity, such an ambitious program is still far from being available.

For this reason I will confine my contribution to the discussion of the specific role of the dust complex for modelling dust forming shells. I place particular emphasis on the global and local shell dynamics and energies, viewed by a sequence of decreasing characteristic scale lengths $l_{\text {mod }}>l_{\text {meso }}>l_{\text {small }}$, each inherently defining a specific scope of approach referring to the global behaviour of dusty shells, spatial instability or mesoturbulence, and effects of small scale fluctuations, respectively (see Fig. 1). 


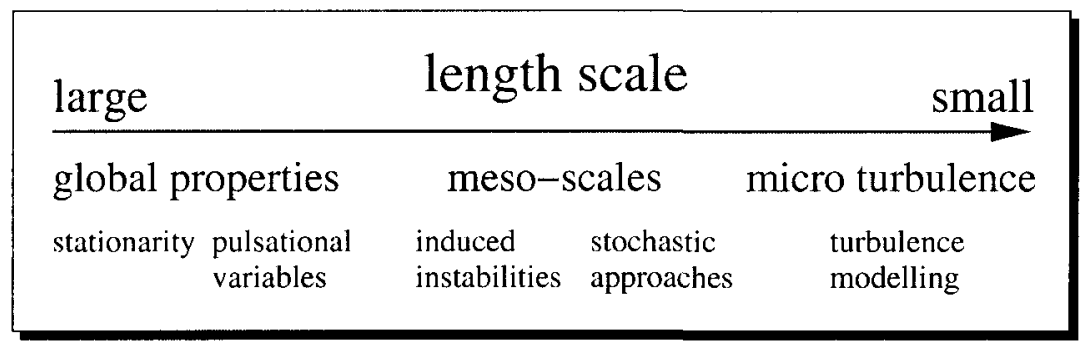

Figure 1. Sequence of characteristic scale length.

\section{The role of dust in the shells of red giants, Miras and LPVs}

For all systems exhibiting effective grain formation, the emergence of dust has a decisive impact upon their local and global structure due to the very effective conversion of radiative energy to longer wavelengths and of radiative momentum to effective dust and gas motions. This usually results in pronounced spectral IR-excesses and in large-scale velocity fields, giving rise to dust-driven winds (e.g., stationary substantial mass loss) or, in the case of pulsating objects, to an extremely nonlinear nonmonotonic wind structure dominated by dust-triggered shock waves and often also by a variety of dust-induced instabilities on different time and length scales.

With regard to statistics, due to the observed flux ratio $F_{\nu}(12 \mu \mathrm{m}) / F_{\nu}(2 \mu \mathrm{m})$, which is considered to be particularly sensitive to the occurrence of dust in the shell of an object, about $20 \%$ of the heavily dust enshrouded stars obviously indicate no variability, and hence show basically a stable mass loss. The other $80 \%$ are pulsational variables (Miras and LPVs), exhibiting a complicated dynamical wind structure (Jura 1986).

Here we are mainly interested in the dynamical and energetic effects induced by dust formation, caused by the coupling of the dust complex to the hydrodynamical and to the energy equations governing the shell structure, and not directly in the chemical nature and the physical description of the dustformation process itself. Irrespective of the chemical details of dust formation, these effects depend mainly on the absorption, scattering and emission properties of the present dust component. Nevertheless, any realistic quantitative modelling has to be based on a reliable dust formation ab initio theory and an adequate chemical treatment of the considered system. But since the equation of motion only requires a reliable dust extinction coefficient to provide a proper acceleration term and the energy equation needs only an appropriate dust absorption, and emission coefficient for describing the local temperature balance, the dynamical and energetic behaviour of a dust shell is essentially determined by the fact that a critical amount of dust is present (i.e. critical optical depth), but not so much on the specific chemical nature of the dust grains.

However, the basic processes always have to be incorporated in any reliable description of the overall system. The specific definition of the particular processes taken into account depends on the adopted basic assumptions about geometry, dynamics, chemistry, etc. Due to the various couplings, the result- 
ing system of fundamental equations becomes strongly nonlinear. The solution may result in different classes of stationary or dynamical models, showing global or local stability or instability governed by the prescribed initial and boundary conditions, but most essentially on the adopted "window of perspective" for the approach chosen, which is strongly depending on the specific scale length to be considered as essential for the intended model.

\subsection{The global perspective}

In this view, we are interested in the modelling of the basic structures of a stellar dust shell, being the result of a consistent description that provides in a certain physical approximation of the local phenomena a realistic quantitative representation of the large scale features of the considered object, like its mass loss rate, its general wind structure, its overall dust production, its spectral appearance, etc.

Typical for this type of approach, where the entire dust shell is envisaged by choosing $l_{\text {mod }} \simeq$ geometrical extension of the shell, are the models of dustdriven winds of giants and supergiants, and also the atmospheres and shells of pulsating AGB stars, as Miras and LPVs.

Stationary dust-driven mass loss: Red giants and supergiants are able to exhibit dust driven mass loss for long time periods. The resulting large mass-loss rate will usually have a significant impact upon stellar late type evolution, in particular for objects on the AGB. In general, dust-driven winds only exist for objects situated in the upper right region of the HR-diagram and thus are confined to stars having low stellar mass $\left(M_{*}<2 M_{\odot}\right)$, very high luminosity $\left(L_{*}>10^{4} L_{\odot}\right)$, and low effective temperature $\left(T_{*}<2500 \mathrm{~K}\right)$, defining in the HRdiagram a limit line of minimum mass-loss rate for objects to be able to develop a stable dust driven wind. All consistent models are completely determined by the prescription of four fundamental external parameters: effective temperature $T_{*}$, stellar luminosity $L_{*}$, stellar mass $M_{*}$, and the set of chemical abundances $\left\{\ldots, \epsilon_{i}, \ldots\right\}$ of the chosen initial element composition, which are necessary and sufficient to fully characterise the object (e.g., Winters et al., 1994).

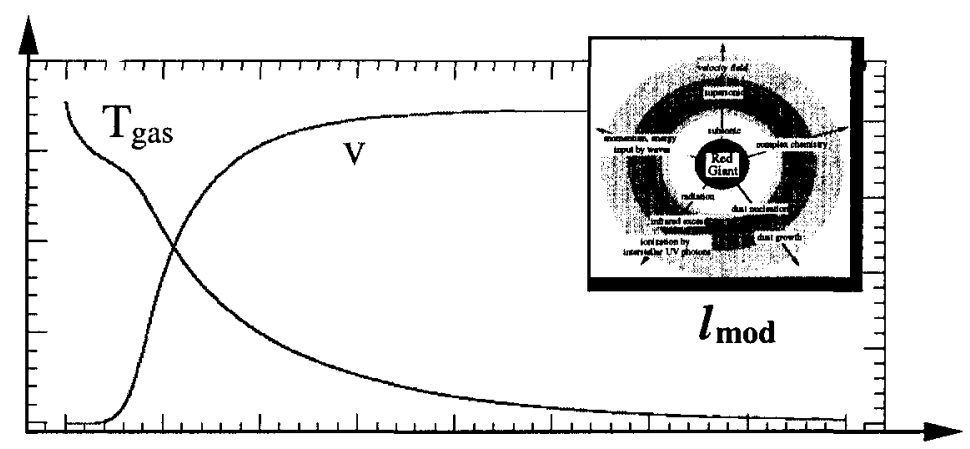

Figure 2. Sketch of the usually adopted "window of perception" showing typical results of the hydrodynamical and thermodynamical structure of a stationary wind. 
For the existence of any real dust-driven wind, i.e., where the emerging dust components are responsible for the wind generation and hence determine the mass loss rate $\dot{M}$ of the star, it is a necessary condition that already sufficient dust has formed inside the radius of the critical velocity of the flow (usually the sonic point), for the radiative acceleration of dust being able to initiate the subsonic-supersonic transition of the velocity field and subsequently to surmount the escape point (Fig. 2). This (first order) global stability is maintained by the action of a stabilising control cycle, resulting in total in a negative feed back, which causes an effective damping of emerging global scale instabilities (cf. Sedlmayr, 1994).

Pulsating atmospheres: This case, which concerns grain condensation in the pulsating shells of Miras and LPVs, addresses the most frequent situation of circumstellar dust formation. In all systems the condensation process is strongly connected with the shock wave structure of the shell, usually triggered by a 2step mechanism. First we have avalanche local nucleation of critical clusters initiated by a passing shock wave, and then efficient growth to macroscopic dust grains behind a following shock, finally resulting in a basically layered structure of the circumstellar dust shell. Due to the strong coupling between the dust component and the radiation field, the structure has a significant influence on both the detailed time-dependent spectral appearance (see, for instance, Winters et al., 1995) and the local and global shell dynamics e.g., Fleischer et al., 1992).

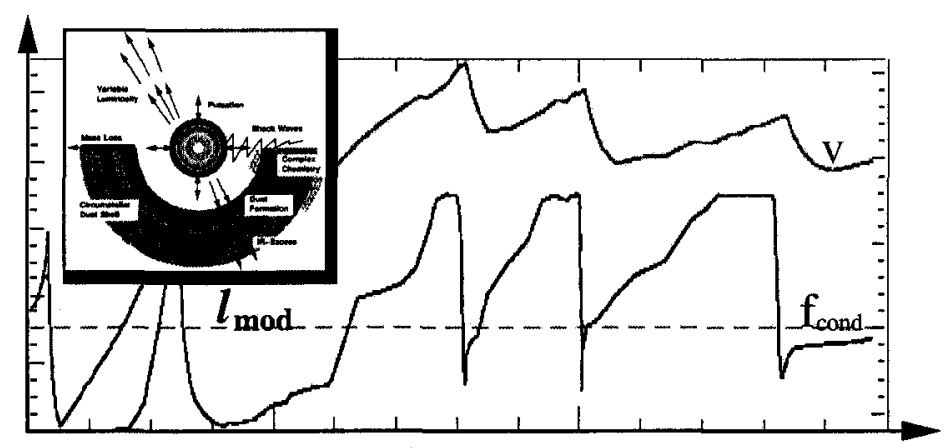

Figure 3. Schematic illustration of the "global perspective" on pulsating circumstellar dust shells depicting typical results of the hydrodynamical structure and dust distribution.

As a cause for the fundamental stellar pulsations, a $\kappa$-mechanism is assumed which determines the dynamics of the stellar envelope and which gives rise to more or less periodical waves travelling outwards through the stellar atmosphere and the extended shell. However, until now no consistent physical modelling of this pulsation mechanism for Miras and LPVs is available (cf. Dorfi \& Feuchtinger, 1991). For this reason, in hitherto model calculations of circumstellar shells the inner pulsation of such objects is simulated by adopting a suitable boundary condition at the base of the stellar atmosphere (piston approximation) in order to account for the underlying pulsational dynamics of the star. Thus, in comparison to stationary dust-driven winds, two additional 
external parameters, namely the fundamental period of the stellar pulsation and the corresponding velocity amplitude, have to be specified for a complete determination of a dynamical wind model.

Initiated by this oscillating piston at the base of the atmosphere, waves are generated which travel outwards through a medium with decreasing density and temperature, thus steepening up and finally evolving a characteristic pattern of supersonic shocks. These produce pronounced local velocity, temperature, and density jumps and, behind the shock fronts, regions of increased density and effective cooling, favourable for cluster nucleation and grain growth. The detailed dynamics of the evolving expanding layered dust shell is controlled by a variety of physical and chemical time scales, giving rise to a complex nonlinear interplay among the various processes. These finally result in an additional exterior $\kappa$-mechanism governing the large scale dynamical behaviours of the circumstellar dust shell.

The dynamics of the models due to the exterior $\kappa$-mechanism reveal an eigenperiod, which is the characteristic time scale between two dust-induced shocks. In numerical models for carbon rich shells, this eigenperiod was found to depend nonlinearly on the luminosity and to be anti-correlated with the overabundance of carbon-to-oxygen, determining the grain formation. Therefore, the actual dynamics of the shell depends not only on the prescribed piston parameters (inner boundary condition) but also significantly on the fundamental stellar parameters, in particular on the chemical abundances which determine the dust forming species. In view of dynamical processes, these abundances in carbon-rich situations the $\mathrm{C} / \mathrm{O}$-ratio - seem to have the role of a "control parameter" for the basic dynamical behaviour of the pulsating shell. Numerical calculations for C-Miras and LPVs show that, by variation of the C/O-ratio, monoperiodic, double periodic, multi-periodic and even chaotic variations of the shell are generated (e.g., Fleischer et al., 1995).

\subsection{Meso-scale perspectives}

In this "window of perception" we focus on physical processes or structures determining the dynamics and actual appearance of a circumstellar dust shell in the regime of length scales considerably smaller than its global dimension but definitely larger than those of local phenomena such as the small scale turbulence regime or the thermal limit (cf. Fig. 4). Such meso-scale phenomena can, for example, be induced by thermodynamical, hydrodynamical or radiative signatures of relevant transport processes, conducted by evolving instabilities or by extended fluctuations of certain physical quantities due to spatial correlations of a turbulent velocity field.

Meso-scale structures due to instabilities: Dust-forming systems are characterized by the presence of important internal thermo-kinetic, dynamical, and radiative feedbacks. Such non-linear systems are well known to be capable of hosting various instabilities, which can provoke the formation of spatio-temporal structures on meso scales.

In many situations encountered in pulsating circumstellar dust-forming shells, instabilities occur giving rise to large scale structure formation, the typical dimension of which is of the order of the characteristic length scales imposed 


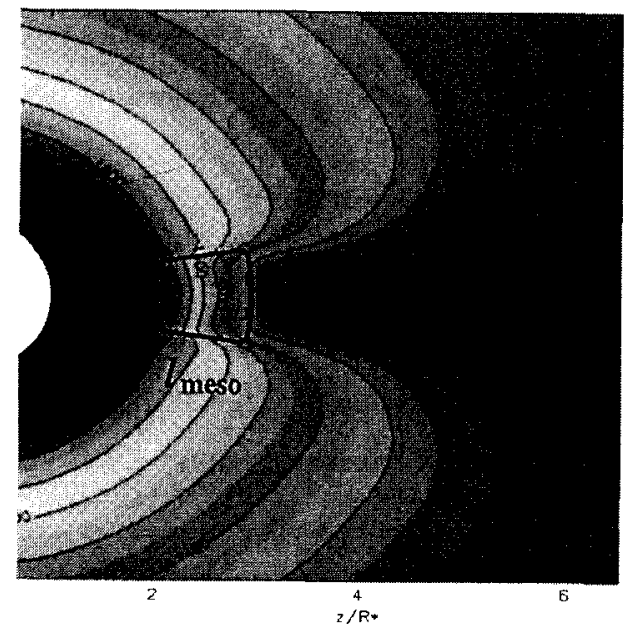

Figure 4. Structures on meso-scales as illustrated here by a dust cloud casting a shadow (Woitke, private communication).

by processes relevant for the dynamics or thermodynamics of the system. With regard to pulsating circumstellar dust shells, three control cycles have been identified and studied in view of macroscopic structure formation in particular by the work of P. Woitke. These cycles concern i) dust induced dynamical instabilities, ii) thermal bifurcations due to molecule formation, and iii) radiative instabilities connected to efficient dust condensation (see Woitke, 2001 and references therein).

One of these instabilities arises from the strong radiative acceleration of dust grains in luminous objects, which may lead to an unstable dynamical behaviour of the dust-forming gas: dust-induced shock waves (see Fig. 5). An additional compression of the gas enhances the chemical reaction rates responsible for nucleation and growth and hence accelerates the dust-formation process. The respective faster increase of the opacity causes a stronger acceleration of the dust-forming zones by radiation pressure, which compresses the gas situated behind these zones. Thus, an overall positive feedback is given, i.e., a selfamplifying control loop which intensifies initial perturbations in the system. The latter, non-local effect of acceleration and compression leads to a wave-like propagation of these disturbances, finally resulting in the formation of strong shock waves. A radial structuring of the dust-forming gas in circumstellar envelopes results, which can produce dust shells even in models without stellar pulsation.

Another instability can occur in partly molecular gases: thermal bifurcations. Molecules strongly contribute to the radiative cooling because of their large number of permitted ro-vibrational transitions in the infrared and microwave spectral region. As a consequence of this cooling, the thermal conditions for subsequent molecule formation are improved (see Fig. 6). This thermal instability leads to the existence of two major types of radiative equilibrium solutions, which can principally co-exist side by side: a low-temperature, molecule-rich so- 


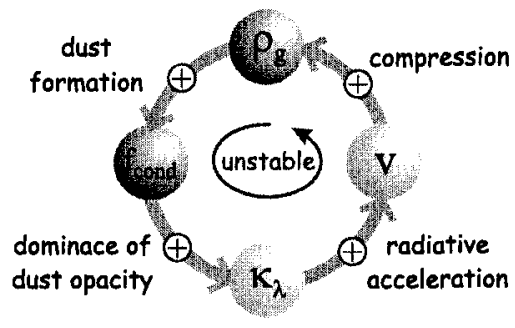

Figure 5. Dust induced dynamical instability.

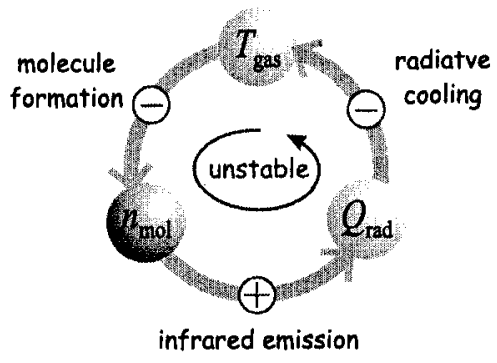

Figure 6. Thermal instability in partially molecular gases.

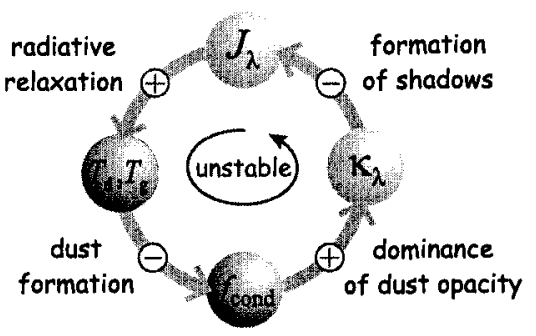

Figure 7. Radiative instability of dust formation.

lution and a high-temperature molecule-poor solution. Both phases are stable and in radiative equilibrium for not too strong and not too weak radiation fields. This bifurcation is possibly involved in the onset of the formation of dust clouds around warm and hot stars, e.g., R CrB and WR stars, where the radiation field can maintain both solutions.

A third instability in dust-forming, irradiated gases results from radiative transfer effects. A local increase of the opacity due to dust formation affects the radiation field in various ways. One effect addressed in Fig. 7 is the shielding from irradiated photons behind already condensed zones. In the shadow of such a zone, the mean intensity $J_{\nu}$ is reduced and, hence, the gas and dust temperatures decrease. Consequently, the conditions for subsequent dust formation are improved, which again forms a self-amplifying feedback loop. This radiative in- 
stability of dust-forming gases leads to the formation of linear, radially aligned dust structures perpendicular to the preferred direction of the radiative flux.

Meso-turbulent circumstellar dust shells: This type of approach is motivated by observations that many circumstellar shells, e.g., the extended atmospheres of WC stars, red giants and supergiants, exhibit a patchy structure (dust clouds?), which might well be connected with pronounced turbulent motions. Thus, turbulence may impose a network of different lengths scales upon the overall structure of the shell, inducing pronounced spatial fluctuations of the local thermodynamical quantities. Such fluctuations should have a significant influence on chemistry and dust formation in circumstellar shells.

As a first step we focus on temperature fluctuations which, due to the extreme temperature sensitivity of dust nucleation, should have a dominant effect on the resulting spatial dust distribution in the shell, possibly giving rise to pronounced cloudy structures. However, until now no consistent detailed treatment of such a complex problem is at hand.

For this reason, a rather simple kinetical description, usually addressed as meso-turbulence approach (cf. Gail et al., 1976), is applied by assuming a stochastic medium where dust condensation occurs due to a Markovian process. Such a first-order approach is possible because the basic moment equations describing grain nucleation and growth in a co-moving frame are first-order ordinary differential equations with regard to the time coordinate (Gail \& Sedlmayr, 1988), which in case of a monotonic background velocity structure of the stellar wind, can easily be expressed by a corresponding radial coordinate. Thus, in view of spatial fluctuations, this method is confined to basically stationary situations showing meso scale inhomogeneities. In this approach, dust formation is conceived as a stochastic process in the expanding shell controlled by the statistical fluctuations, in particular of the local temperature. This description yields local expectation values for the relevant moments describing dust formation, which depend on two external parameters characterizing the stochastic behaviour of the adopted thermal quantity: the width $\sigma$ of the 1-point distribution function and a transition length $l$ of the corresponding transition probability in the resulting system of Fokker-Planck equations (cf. Gail et al., 1980). By demonstrative applications of this method to dust-driven winds with a fluctuating temperature, a significant influence on the local dust distribution in the meso scale regime has been found, in good agreement with detailed observations: First, the inner edge of the circumstellar dust shell compared to deterministic calculations seems to be shifted significantly nearer to the stellar surface, and second, the probability regime of efficient dust formation is considerably extended to objects having larger effective temperatures (Dirks, 2000).

\subsection{Small-scale structures}

This situation is exhibited by the small-scale limit of a turbulent situation, resulting from decaying turbulence in systems characterized by high Reynolds numbers, like stellar atmospheres and circumstellar shells. In view of applying turbulence theory to dust formation, only atmospheres of brown dwarfs have been investigated so far by adopting the small-scale limit $l_{\text {mod }} \gg l_{\text {meso }} \gg$ $l_{\text {small }} \sim 10 \mathrm{~m} \gg l_{\text {kin }}$. The results of Helling et al. (2001), obtained by the mod- 


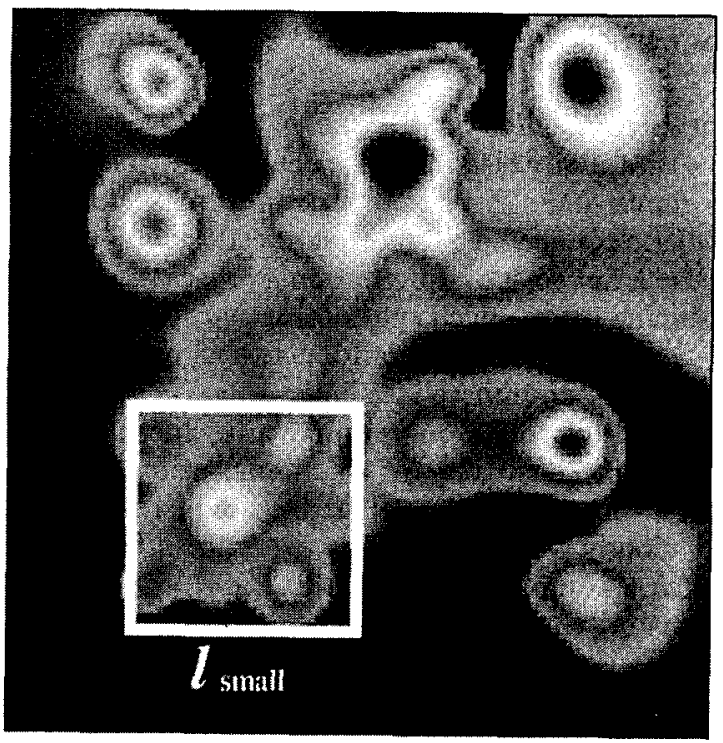

Figure 8. Inhomogeneous distribution of the dust particle number density due to small scale turbulence.

elling of turbulent hydrodynamics, thermodynamics and dust condensation for a chosen volume of mass, indicate effective dust formation in evolving local inhomogeneities due to small-scale turbulence, yielding a finely structured spatial dust distribution (cf. Fig. 8). The models to date, however, are intended to describe quantitatively the asymptotic situation of turbulence, providing the basis for their future extension to intermediate and large scales, which is necessary for a consistent complete numerical modelling of a turbulent dust-forming stellar atmosphere.

\section{Concluding remarks}

Viewing an object within a particular range of scale lengths requires not only adequate observations and theories, but also projects specific properties, not revealed by choosing other perspectives. All are relevant for a real understanding of local and global behaviour and thus have to be brought together to complete the puzzle of a final comprehensive picture. In this regard, even our very elaborate approaches to dust-forming shells are still far from meeting this necessary condition in many essential aspects.

Acknowledgement: I thank very much Dr. B. Patzer and Dr. P. Woitke for their assistance preparing the manuscript. 


\section{References}

Dirks, U. 2000, Doktorthesis, TU Berlin

Dorfi, E.A. \& Feuchtinger M.U. A\&A, 249, 417

Fleischer, A.J., Gauger, A., \& Sedlmayr E. 1992, A\&A, 266, 321

Fleischer, A.J., Gauger, A., \& Sedlmayr E. 1995, A\&A, 297, 543

Gail, H.-P., Sedlmayr, E., \& Traving, G. 1976, A\&A, 46, 441

Gail, H.-P., Sedlmayr, E., \& Traving, G. 1980, JQSRT, 23, 267

Gail, H.-P. \& Sedlmayr, E. 1988, A\&A, 206, 153

Helling, Ch., Oevermann, M., Lüttke, M., Klein, R., \& Sedlmayr, E. 2001, A\&A, 376,194

Jura, M. 1986, ApJ, 303, 327

Sedlmayr, E. 1994, in Lecture Notes in Physics, Vol. 428, Molecules in the Stellar Environment, ed. U. Jørgensen, (Springer-Verlag Berlin), 163

Winters, J.M., Dominik, C., Sedlmayr, E. 1994, A\&A, 288, 255

Winters, J.M., Fleischer, A. J., Gauger, A., \& Sedlmayr, E. 1995, A\&A, 302, 483

Woitke, P. 2001, Rev. Mod. Astr., 14, 185 